\title{
CHECKING THE STRICT POSITIVITY OF KRAUS MAPS IS NP-HARD
}

\author{
STÉPHANE GAUBERT AND ZHENG QU
}

\begin{abstract}
Basic properties in Perron-Frobenius theory are strict positivity, primitivity, and irreducibility. Whereas for nonnegative matrices, these properties are equivalent to elementary graph properties which can be checked in polynomial time, we show that for Kraus maps - the noncommutative generalization of stochastic matrices - checking strict positivity (whether the map sends the cone to its interior) is NP-hard. The proof proceeds by reducing to the latter problem the existence of a non-zero solution of a special system of bilinear equations. The complexity of irreducibility and primitivity is also discussed in the noncommutative setting.

KEYWORDS. Perron-Frobenius theory, multilinear algebra, computational complexity, positive dynamical systems, noncommutative Markov chains, noncommutative consensus, completely positive maps, quantum control and information theory.
\end{abstract}

\section{INTRODUCTION}

Irreducibility, primitivity, and strict positivity are basic structural notions of Perron-Frobenius theory [BP94]. Recall that a linear map $A$ leaving invariant a (closed, convex, and pointed) cone $C$ of a vector space is said to be strictly positive if it sends the cone to its interior; primitive if it has a power that is positive, and irreducible if it does not leave invariant a non-trivial face of the cone. These notions allow one to determine spectral or dynamical properties of the map. In particular, the strongest of the above notions, strict positivity, entails the strict contraction of $A$ with respect to Hilbert's projective metric (Birkhoff's theorem), and so, the convergence of the rescaled iterates of $A$ to a rank one linear map with a geometric rate. The latter property is of importance in a number of applications, including "consensus theory" for distributed systems or population dynamics. It is natural to ask how properties of this nature can be checked for various classes of cones.

If $C$ is the standard positive cone of $\mathbb{R}^{n}, A$ can be identified to a nonnegative matrix $A \in M_{n}(\mathbb{R})$. Then, strict positivity, primitivity, and irreducibility, can be easily checked. Indeed, a nonnegative matrix $A$ is strictly positive if and only if all its entries are positive. Moreover, $A$ is primitive if and only if $A^{n^{2}-2 n+2}$ is strictly positive [HJ13]. Finally, it is irreducible if and only if the associated directed graph is strongly connected. Note also that an efficient combinatorial algorithm is available to compute the period of an irreducible matrix, which allows one in particular to decide if it is primitive [Den77]. Therefore, primitivity and irreducibility for nonnegative matrices are equivalent to well known problems of graph theory, that can be solved in polynomial time.

Another important class of maps arises when considering the cone $C$ of positive semidefinite matrices. Then, the noncommutative analogue of a stochastic matrix is a Kraus map, i.e., a completely positive and trace-preserving map on this cone. Kraus maps are fundamental objects in quantum control and information theory, as they represent quantum channels. The notions of irreducibility, strict positivity and primitivity are of importance for Kraus maps, see in particular [Far96, SPGWC10, SSR10, RKW11]. It is natural to ask whether we can verify these properties for Kraus maps in polynomial time, as in the case of nonnegative matrices.

Our main result, Theorem 4.2 asserts that checking the strict positivity of a Kraus map is NP-hard. It may come as a surprise that strict positivity, which is the simplest property in the case of nonnegative matrices, turns out to be the hardest one in the case of Kraus maps. Indeed, we derive from previous results that the irreducibility and primitivity of a Kraus map can be checked in polynomial time. A classical lemma of Burnside on matrix algebras combined with a result of Farenick [Far96] implies that the

This work was partially supported by the PGMO (Gaspard Monge) Program of FMJH (Fondation Mathématique Jacques Hadamard) and EDF. It was carried out when the second author was with CMAP, Ecole Polytechnique and INRIA, being supported by a doctoral fellowship of Ecole Polytechnique. 
irreducibility of a completely positive map can be checked in polynomial time. Moreover, a characterization given by Sanz, Pérez-García, Wolf and Cirac [SPGWC10] also implies that the primitivity of a Kraus map can be checked in polynomial time. See Corollary 3.1 below for the derivation of these two facts. Note that in each of these results, we assume that the input -which determines the Kraus mapconsists of the Kraus operators.

To show Theorem 4.2, we first show that the strict positivity of a Kraus map is equivalent to the non feasibility of the bilinear system given by the Kraus operators, or equivalently the non-existence of a rank one matrix in the orthogonal complement of the subspace generated by the Kraus operators, see Lemma 4.1 Then, we prove that every 3SAT problem can be reduced in polynomial time to the problem of checking the feasibility of a bilinear system given by a set of Kraus operators, see Theorem 4.1

We note that several rank minimization problems have been extensively studied in the literature [FHB04, RXH11, FSEDS13]. In particular, the problem of finding a matrix of minimal rank in a affine subspace is known to be NP-hard [BFS99, RFP10, DTDS12] and hard to approximate [Nat95]. However, here the matrix subspace is linear instead of affine, and rank minimization in a linear subspace is a trivial subproblem. Note also that Hillar and Lim [HL13] showed the NP-hardness of the bilinear feasibility problem, by reducing the graph 3-Colorability problem to it. However, the bilinear systems arising from a Kraus map are special due to the unital constraint or trace-preserving property of the Kraus map. Hence Theorem 4.1 is a different result; it does not seem easy to deduce it from the NP-hardness of checking the feasibility of bilinear systems, see Remark 4.1 .

\section{IRREDUCIBILITY, PRIMITIVITY AND STRICT POSITIVITY FOR COMPLETELY POSITIVE MAPS}

Throughout the paper, the space of Hermitian matrices is denoted by $S_{n}$. Denote by $\preccurlyeq(\prec)$ the (strict) Loewner order on the space $\mathrm{S}_{n}$, i.e., $A \preccurlyeq B(A \prec B)$ if and only if $B-A$ is a positive semidefinite (definite) matrix. The adjoint matrix (conjugate transpose) of a matrix $A \in \mathbb{C}^{n \times n}$ is denoted by $A^{*}$. $\mathrm{S}_{n}$,

To a family of $n \times n$ complex matrices $V_{1}, \ldots, V_{m}$, we associate the completely positive map $\Psi: \mathrm{S}_{n} \rightarrow$

$$
\Psi(X):=\sum_{i=1}^{m} V_{i} X V_{i}^{*}, \quad X \in \mathrm{S}_{n} .
$$

This map is said to be a Kraus map if

$$
\sum_{i=1}^{m} V_{i}^{*} V_{i}=I_{n}
$$

then, the matrices $V_{1}, \ldots, V_{m}$ are called Kraus operators.

We denote by $\mathscr{S}_{k}\left(V_{1}, \ldots, V_{m}\right)$ the complex linear space spanned by all the products of $k$ Kraus operators $\left\{V_{1}, \ldots, V_{m}\right\}$ :

$$
\mathscr{S}_{k}\left(V_{1}, \ldots, V_{m}\right):=\operatorname{span}\left\{V_{i_{k}} \ldots V_{i_{1}}: i_{k}, \ldots, i_{1} \in\{1, \ldots, m\}\right\} .
$$

We also denote by $\mathscr{D}_{k}\left(V_{1}, \ldots, V_{m}\right)$ the complex linear space spanned by all the products of at most $k$ Kraus operators:

$$
\mathscr{D}_{k}\left(V_{1}, \ldots, V_{m}\right):=\operatorname{span}\left\{V_{i_{j}} \ldots V_{i_{1}}: 1 \leqslant j \leqslant k, i_{j}, \ldots, i_{1} \in\{1, \ldots, m\}\right\} .
$$

We denote by $\mathscr{A}\left(V_{1}, \ldots, V_{m}\right)=\cup_{k \geqslant 1} \mathscr{D}_{k}\left(V_{1}, \ldots, V_{m}\right)$ the algebra generated by the Kraus operators $\left\{V_{1}, \ldots, V_{m}\right\}$ :

$$
\mathscr{A}\left(V_{1}, \ldots, V_{m}\right):=\operatorname{span}\left\{V_{i_{k}} \ldots V_{i_{1}}: k \in \mathbb{N}, i_{k}, \ldots, i_{1} \in\{1, \ldots, m\}\right\} .
$$

Lemma 2.1. There is $p \leqslant n^{2}$ such that $\mathscr{A}\left(V_{1}, \ldots, V_{m}\right)=\mathscr{D}_{p}\left(V_{1}, \ldots, V_{m}\right)$.

Proof. It is clear that for all $k=1,2, \ldots$, we have

$$
\mathscr{D}_{k+1}\left(V_{1}, \ldots, V_{m}\right) \supset \mathscr{D}_{k}\left(V_{1}, \ldots, V_{m}\right) \cup\left\{V_{i} X: X \in \mathscr{D}_{k}\left(V_{1}, \ldots, V_{m}\right), i \in\{1, \ldots, m\}\right\} .
$$

Hence there is $p \leqslant n^{2}$ such that $\mathscr{D}_{p+1}\left(V_{1}, \ldots, V_{m}\right)=\mathscr{D}_{p}\left(V_{1}, \ldots, V_{m}\right)$ and thus $\mathscr{A}\left(V_{1}, \ldots, V_{m}\right)=\mathscr{D}_{p}\left(V_{1}, \ldots, V_{m}\right)$.

We next recall the definitions of irreducibility, strict positivity and primitivity for completely positive maps. 
Definition 2.1 (Irreducibility [Far96]). The map $\Psi$ is irreducible if there is no face of $\mathrm{S}_{n}^{+}$invariant by $\Psi$, where a face $\mathscr{F}$ of $\mathrm{S}_{n}^{+}$is a (closed, convex) cone strictly contained in $\mathrm{S}_{n}^{+}$such that if $P \in \mathscr{F}$ then $Q \in \mathscr{F}$ for all $Q \preccurlyeq P$.

Definition 2.2 (Strict positivity). The map $\Psi$ is strictly positive if for all $X \succcurlyeq 0, \Psi(X) \succ 0$.

A standard compactness argument shows that $\Psi$ is strictly positive if and only if

$$
\Psi(X) \succcurlyeq \alpha \operatorname{tr}(X) I, \quad \forall X \in \mathrm{S}_{n}^{+}
$$

for some constant $\alpha>0$.

Definition 2.3 (Primitivity [SPGWC10]). The map $\Psi$ is primitive if there is an integer $p>0$ such that $\Psi^{p}$ is strictly positive.

It will be convenient to consider the following three problems.

Problem 2.1 (Irreducibility of Completely Positive Maps). Input: integers $n, m$, and matrices $V_{1}, \ldots, V_{m} \subset$ $\mathbb{C}^{n \times n}$ with rational entries.

Question: Is the map $\Psi$ defined by (1) irreducible?

Problem 2.2 (Primitivity of Completely Positive Maps). Input: integers $n, m$, and matrices $V_{1}, \ldots, V_{m} \subset$ $\mathbb{C}^{n \times n}$ with rational entries

Question: Is the map $\Psi$ defined by (1) primitive?

Problem 2.3 (Strict positivity of Kraus maps). Input: integers $n, m$, and matrices $V_{1}, \ldots, V_{m} \subset \mathbb{C}^{n \times n}$ with rational entries, satisfying

$$
\sum_{i=1}^{m} V_{i}^{*} V_{i}=I_{n}
$$

Question: Is the Kraus map associated to $\left\{V_{1}, \ldots, V_{m}\right\}$ strictly positive?

We next show that the first two problems can be solved in polynomial time whereas the last one is NP-hard.

\section{CHECKING THE IRREDUCIBILITY AND PRIMITIVITY IS POLYNOMIAL}

We shall need the following characterization of irreducibility.

Proposition 3.1. The completely positive map $\Psi$ given by (1) is irreducible if and only if $\mathscr{A}\left(V_{1}, \ldots, V_{m}\right)=$ $\mathbb{C}^{n \times n}$.

Proof. Farenick showed in [Far96, Theorem 2] that the reducibility of $\Psi$ is equivalent to the existence of a non-trivial (other than $\{0\}$ or $\mathbb{C}^{n}$ ) common invariant subspace of all $\left\{V_{i}\right\}$. By Burnside's theorem on matrix algebra (see [LR04]), the latter property holds if and only if the algebra $\mathscr{A}\left(V_{1}, \ldots, V_{m}\right)$ is not the whole matrix space.

We shall need the following characterization of primitivity of completely positive maps, which is a consequence of a "quantum version of Wielandt inequality" established by Sanz, Pérez-García, Wolf and Cirac for Kraus maps.

Theorem 3.1 (Corollary of [SPGWC10] $)$. Assume that the completely positive map $\Psi$ is irreducible. Then, $\Psi$ is primitive if and only if there is $q \leqslant\left(n^{2}-m+1\right) n^{2}$ such that the space $\mathscr{S}_{q}\left(V_{1}, \ldots, V_{m}\right)$ coincides with $\mathbb{C}^{n \times n}$, for some $q \leqslant\left(n^{2}-m+1\right) n^{2}$.

Proof. Theorem 1 of [SPGWC10] shows that if $\Psi$ is a Kraus map, then, it is primitive if and only if $\mathscr{S}_{q}\left(V_{1}, \ldots, V_{m}\right)$ coincides with $\mathbb{C}^{n \times n}$, for some $q \leqslant\left(n^{2}-m+1\right) n^{2}$. We next show that this implies that the same property holds for all irreducible completely positive maps. Indeed, it follows from the PerronFrobenius theorem that the adjoint map $\Psi^{*}$ has an eigenvector $A$ in the cone of positive semidefinite matrices such that the associated eigenvalue is the spectral radius of $\Psi, \rho(\Psi)$, i.e.

$$
\sum_{1 \leqslant i \leqslant m} V_{i}^{*} A V_{i}=\rho(\Psi) A
$$


Since $\Psi$ is irreducible, $\Psi^{*}$ is also irreducible (this follows from [Far96, Theorem 2]), and so this eigenvector must belong to the interior of the cone, meaning that $A$ is a positive definite matrix. Now, for all invertible matrices $U$, define $\Gamma_{U}(X):=U X U^{*}$. Then, the map $\Phi=\rho(\Psi)^{-1} \Gamma_{A^{1 / 2}} \circ \Psi \circ \Gamma_{A^{-1 / 2}}$ satisfies

$$
\Phi(X)=\sum_{i=1}^{m} W_{i} X W_{i}^{*}, \quad \text { with } W_{i}=\rho(\Psi)^{-1 / 2} A^{1 / 2} U_{i} A^{-1 / 2},
$$

and it follows from (4) that it is a Kraus map. Moreover, since $\mathscr{S}_{q}\left(V_{1}, \ldots, V_{m}\right)=A^{-1 / 2} \mathscr{S}_{q}\left(W_{1}, \ldots, W_{m}\right) A^{1 / 2}$, $\mathscr{S}_{q}\left(V_{1}, \ldots, V_{m}\right)$ coincides with $\mathbb{C}^{n \times n}$ if and only if $\mathscr{S}_{q}\left(W_{1}, \ldots, W_{m}\right)$ does.

Corollary 3.1. The irreducibility and the primitivity of a completely positive map can be checked in polynomial time.

Proof. By Proposition 3.1 and Lemma2.1, to decide if the Kraus map $\Psi$ is irreducible, we shall compute the increasing sequence of matrix subspaces $\mathscr{D}_{s}\left(V_{1}, \ldots, V_{m}\right), s=1,2, \ldots$, and look for the first integer $k \leqslant n^{2}$ such that $\mathscr{D}_{k}\left(V_{1}, \ldots, V_{m}\right)=\mathscr{D}_{k+1}\left(V_{1}, \ldots, V_{m}\right)$. For a given $s$, we shall represent $\mathscr{D}_{s}\left(V_{1}, \ldots, V_{m}\right)$ by a basis, i.e.,

$$
\mathscr{D}_{s}\left(V_{1}, \ldots, V_{m}\right)=\operatorname{span}\left\{M_{1}, \cdots, M_{l}\right\}
$$

where $\left\{M_{1}, \ldots, M_{l}\right\} \in \mathbb{C}^{n \times n}$ are linearly independent matrices. Recall that extracting a basis from a family of rational vectors can be done in polynomial time in the bit model. Since $\mathscr{D}_{s+1}\left(V_{1}, \ldots, V_{m}\right)=$ $\operatorname{span}\left\{V_{i} M_{s}, M_{s}, 1 \leqslant i \leqslant m, 1 \leqslant s \leqslant l\right\}$, it follows that we can compute inductively a basis $M_{1}, \cdots, M_{l}$ of $\mathscr{D}_{s}\left(V_{1}, \ldots, V_{m}\right)$, with $l \leqslant n^{2}$, and that the number of bits needed to code the basis elements $M_{1}, \ldots, M_{l}$ remain polynomially bounded in the length of the input. Hence, a basis representation of the algebra $\mathscr{A}\left(V_{1}, \ldots, V_{m}\right)$ can be obtained in polynomial time.

Arguing as above, a basis representation of $\mathscr{S}_{q}\left(V_{1}, \ldots, V_{m}\right)$ for some $q \leqslant\left(n^{2}-m+1\right) n^{2}$ can be computed in polynomial time. Thus, to check the primitivity, we first check the irreducibility (which is a necessary condition), and if it is satisfied, we check the condition of Theorem 3.1

\section{CHECKING THE STRICT POSITIVITY IS NP-HARD}

In this section, we study the complexity of Problem 2.3. deciding if a Kraus map is strictly positive. First we show that the strict positivity of a Kraus map is equivalent to the non-existence of rank one matrix in the orthogonal complement of the subspace spanned by the Kraus operators.

Lemma 4.1. The Kraus map $\Psi$ is strictly positive if and only if we cannot find two nonzero vectors $x, y \in \mathbb{C}^{n}$ such that

$$
x^{*} V_{i} y=0, \forall i=1, \ldots, m .
$$

Proof. By definition, the map $\Psi$ is strictly positive if and only if for all nonzero vectors $y \in \mathbb{C}^{n}$, the matrix

$$
\Psi\left(y y^{*}\right)=\sum_{i=1}^{m} V_{i} y y^{*} V_{i}^{*}
$$

is positive definite. This holds if and only if for all nonzero vectors $x \in \mathbb{C}^{n}$,

$$
\sum_{i=1}^{m} x^{*} V_{i} y y^{*} V_{i}^{*} x=\sum_{i=1}^{n}\left|x^{*} V_{i} y\right|^{2}>0
$$

Therefore $\Phi$ is not strictly positive if and only if we can find nonzero vectors $x, y \in \mathbb{C}^{n}$ such that (5) holds.

Hence, the strict positivity of a Kraus map (Problem 2.3) is equivalent to the non feasibility of the following bilinear system associated to the Kraus operators $\left\{V_{1}, \ldots, V_{m}\right\}$.

Problem 4.1 (Unital bilinear feasibility). Input: integers $n, m$, and matrices $V_{1}, \ldots, V_{m} \subset \mathbb{C}^{n \times n}$ with rational entries, satisfying (3). Question: is there a nonzero solution to the following bilinear system:

$$
x^{T} V_{i} y=0, \forall i=1, \ldots, m ?
$$

Problem 4.1 is trivially equivalent to the following problem on the existence of a rank one matrix in the orthogonal complement of the subspace generated by the Kraus operators $\left\{V_{1}, \ldots, V_{m}\right\}$. 
Problem 4.2 (Existence of rank one matrix). Input: integers $n, m$, and matrices $V_{1}, \ldots, V_{m} \subset \mathbb{C}^{n \times n}$ with rational entries, satisfying (3). Question: is there a rank one matrix in the orthogonal complement of the subspace spanned by $\left\{V_{1}, \ldots, V_{m}\right\}$ ?

Consider also the following similar problem without the unital constraint on matrices:

Problem 4.3 (Bilinear feasibility). Input: integers $n, m$, and matrices $W_{1}, \ldots, W_{m} \subset \mathbb{C}^{n \times n}$ with rational entries. Question: is there a nonzero solution to the following bilinear system:

$$
x^{T} W_{i} y=0, \forall i=1, \ldots, m ?
$$

Theorem 4.1. The 3SAT problem is reducible in polynomial time to Problem 4.1

The proof is based on the following observation. An instance of the 3SAT problem with $N$ Boolean variables $X_{1}, \ldots, X_{N}$ and $M$ clauses can be coded by a system of polynomial equations in $N$ complex variables $x_{1}, \ldots, x_{N}$,

$$
\left\{\begin{array}{l}
\left(1+p_{i} x_{k_{i}^{1}}\right)\left(1+q_{i} x_{k_{i}^{2}}\right)\left(1+r_{i} x_{k_{i}^{3}}\right)=0, \quad i=1, \cdots, M \\
x_{i}^{2}=1, \quad i=1, \cdots, N
\end{array}\right.
$$

where $k_{i}^{1}, k_{i}^{2}, k_{i}^{3} \in\{1, \ldots, N\}, p_{i}, q_{i}, r_{i} \in\{ \pm 1\}$ and $k_{i}^{1} \neq k_{i}^{2}$ for all $1 \leqslant i \leqslant M$. The Boolean variable $X_{i}$ is true if $x_{i}=1$ and false if $x_{i}=-1$. For instance, the clause $X_{1} \vee \neg X_{2} \vee X_{4}$ corresponds to the polynomial $\left(1-x_{1}\right)\left(1+x_{2}\right)\left(1-x_{4}\right)$ and the clause $\neg X_{6} \vee \neg X_{1} \vee X_{2}$ corresponds to the polynomial $\left(1+x_{6}\right)(1+$ $\left.x_{1}\right)\left(1-x_{2}\right)$.

Therefore, to prove Theorem 4.1, it is sufficient to construct in polynomial time a set of Kraus operators $\left\{V_{1}, \ldots, V_{m}\right\} \subset \mathbb{C}^{n}$ with rational entries satisfying (3), such that there is a solution to (6) if and only if there are two nonzero vectors $x, y \in \mathbb{C}^{n}$ such that ${ }^{‘}[5]$ holds.

We begin by the following basic lemma.

Lemma 4.2. Let $a_{k}(\cdot, \cdot): \mathbb{C}^{n} \times \mathbb{C}^{n} \rightarrow \mathbb{C}, 1 \leqslant k \leqslant M$ be a finite set of bilinear forms. There is a solution $x \in \mathbb{C}^{n}$ to the system

$$
a_{k}(x, x)=0, \quad 1 \leqslant k \leqslant M
$$

if and only if there is a pair of non-zero vectors $x=\left(x_{i}\right)_{1 \leqslant i \leqslant n}, y=\left(y_{i}\right)_{1 \leqslant i \leqslant n} \in \mathbb{C}^{n}$ satisfying the system

$$
\left\{\begin{array}{l}
a_{k}(x, y)=0, \quad 1 \leqslant k \leqslant M \\
x_{i} y_{j}-x_{j} y_{i}=0, \quad 1 \leqslant i<j \leqslant n
\end{array}\right.
$$

Proof. The last equations require that $y$ be proportional to $x$.

The next lemma shows that system (6) can be transformed into a set of homogeneous equations.

Lemma 4.3. Let $N, M \in \mathbb{N}$. Let $\left(k_{i}^{1}\right)_{i},\left(k_{i}^{2}\right)_{i},\left(k_{i}^{3}\right)_{i}$ be three sequences of integers in $\{1, \cdots, N\}$. Let $\left(p_{i}\right)_{i},\left(q_{i}\right)_{i},\left(r_{i}\right)_{i}$ be three sequences of real numbers. Consider the following system of equations on the variables $\left(x_{i}\right)_{1 \leqslant i \leqslant N}$ :

$$
\left\{\begin{array}{l}
\left(1+p_{i} x_{k_{i}^{1}}\right)\left(1+q_{i} x_{k_{i}^{2}}\right)\left(1+r_{i} x_{k_{i}^{3}}\right)=0, \quad i=1, \cdots, M \\
x_{i}^{2}=1, \quad i=1, \cdots, N
\end{array}\right.
$$

The system (8) has a solution $x \in \mathbb{C}^{N}$ if and only if there is a pair of nonzero vectors $x=\left(x_{i}\right)_{0 \leqslant i \leqslant N+2 M}, y=$ $\left(y_{i}\right)_{0 \leqslant i \leqslant N+2 M} \in \mathbb{C}^{N+2 M+1}$ satisfying the following system:

$$
\left\{\begin{array}{l}
\left(x_{0}+p_{i} x_{k_{i}^{1}}+q_{i} x_{k_{i}^{2}}+p_{i} q_{i} x_{N+i}\right) y_{N+M+i}=0, \quad i=1, \cdots, M \\
x_{k_{i}^{1}} y_{k_{i}^{2}}-x_{0} y_{N+i}=0, \quad i=1, \cdots, M \\
\left(x_{0}+r_{i} x_{k_{i}^{3}}-x_{N+M+i}\right) y_{j}=0, \quad i=1, \cdots, M, \quad j=0, \ldots, N+2 M \\
x_{i} y_{i}-x_{0} y_{0}=0, \quad i=1, \cdots, N+M \\
x_{i} y_{j}-x_{j} y_{i}=0, \quad 0 \leqslant i<j \leqslant N+2 M
\end{array}\right.
$$

Proof. A simple rewriting of the system (8) is:

$$
\left\{\begin{array}{l}
\left(1+p_{i} x_{k_{i}^{1}}+q_{i} x_{k_{i}^{2}}+p_{i} q_{i} x_{k_{i}^{1}} x_{k_{i}^{2}}\right)\left(1+r_{i} x_{k_{i}^{3}}\right)=0, \quad i=1, \cdots, M \\
x_{i}^{2}=1, \quad i=1, \cdots, N
\end{array}\right.
$$


By introducing $2 M$ extra variables, denoted by $\left\{x_{N+i}\right\}_{1 \leqslant i \leqslant 2 M}$, to replace the variables $\left\{x_{k_{i}^{1}} x_{k_{i}^{2}}, 1+r_{i} x_{k_{i}^{3}}\right\}_{i \leqslant M}$, we rewrite the system (10) as:

$$
\left\{\begin{array}{l}
\left(1+p_{i} x_{k_{i}^{1}}+q_{i} x_{k_{i}^{2}}+p_{i} q_{i} x_{N+i}\right) x_{N+M+i}=0, \quad i=1, \cdots, M \\
x_{k_{i}^{1}} x_{k_{i}^{2}}-x_{N+i}=0, \quad i=1, \cdots, M \\
1+r_{i} x_{k_{i}^{3}}-x_{N+M+i}=0, \quad i=1, \cdots, M \\
x_{i}^{2}=1, \quad i=1, \cdots, N+M
\end{array}\right.
$$

We next add an extra variable $x_{0}$ to replace the affine term 1 to construct a system of homogeneous polynomial equations of degree 2 :

$$
\left\{\begin{array}{l}
\left(x_{0}+p_{i} x_{k_{i}^{1}}+q_{i} x_{k_{i}^{2}}+p_{i} q_{i} x_{N+i}\right) x_{N+M+i}=0, \quad i=1, \cdots, M \\
x_{k_{i}^{1}} x_{k_{i}^{2}}-x_{0} x_{N+i}=0, \quad i=1, \cdots, M \\
\left(x_{0}+r_{i} x_{k_{i}^{3}}-x_{N+M+i}\right) x_{j}=0, \quad i=1, \cdots, M, \quad j=0, \ldots, N+2 M \\
x_{i}^{2}-x_{0}^{2}=0, \quad i=1, \cdots, N+M
\end{array}\right.
$$

Then that there is a solution to (11) if and only if there is a solution $x=\left(x_{i}\right)_{0 \leqslant i \leqslant N+2 M}$ to (12) such that $x_{0} \neq 0$. By Lemma 4.2, we know that the system (12) has a solution $x=\left(x_{i}\right)_{0 \leqslant i \leqslant N+2 M}$ with $x_{0} \neq 0$ if and only if there is a pair of non-null vectors $x=\left(x_{i}\right)_{0 \leqslant i \leqslant N+2 M}$ and $y=\left(y_{i}\right)_{0 \leqslant i \leqslant N+2 M}$ with $x_{0} y_{0} \neq 0$ satisfying (9).

So far, we proved that there is a solution to $[8$ if and only if there is a pair of nonzero vectors $x, y \in \mathbb{C}^{N+2 M+1}$ satisfying (9) such that $x_{0} y_{0} \neq 0$. We next prove by contradiction that all nonzero pairs of solutions to (9) satisfy $x_{0} y_{0} \neq 0$.

Let $x=\left(x_{i}\right)_{0 \leqslant i \leqslant N+2 M}$ and $y=\left(y_{i}\right)_{0 \leqslant i \leqslant N+2 M}$ be a pair of nonzero solutions to (9) such that $x_{0} y_{0}=0$. Since by the last constraint in (9), $x$ and $y$ are proportional to each other, we know that $x_{0}=y_{0}=0$. Suppose that there is $1 \leqslant i_{0} \leqslant N+M$ such that $x_{i_{0}} \neq 0$, then by the fourth equation of 9 we know that:

$$
x_{i_{0}} y_{i_{0}}=0 \text {, }
$$

thus $y_{i_{0}}=0$. This implies that $y$ is a zero vector because $x$ and $y$ are proportional to each other. Hence $x_{i}=0$ for all $i \leqslant N+M$. Now we apply this condition to the third equation in (9) to obtain:

$$
x_{N+M+i} y_{j}=0, \quad i=1, \ldots, M, \quad j=0, \ldots, N+2 M .
$$

If $x$ is a nonzero vector, necessarily there is $i_{0}$ such that $x_{N+M+i_{0}} \neq 0$, in that case $y$ is a zero vector. Therefore we deduce that for all nonzero solution of $[9]$, it is necessary that $x_{0} y_{0} \neq 0$.

Lemma 4.4. Consider the system (8) in Lemma 4.3 We suppose in addition that $k_{i}^{1} \neq k_{i}^{2}$ for all $1 \leqslant i \leqslant M$ and that $\left(p_{i}\right)_{i},\left(q_{i}\right)_{i},\left(r_{i}\right)_{i}$ are sequences of numbers in $\{ \pm 1\}$. Let $n=N+2 M+1$. There is a finite family of matrices $\left\{V_{i}\right\}_{1 \leqslant i \leqslant m} \subset \mathbb{C}^{n \times n}$ with entries in $\left\{0, \pm 1, \pm \frac{1}{3}\right\}$ such that the system (8) has a solution if and only if there is nonzero solution to the following bilinear system:

$$
x^{T} V_{i} y=0, \quad i=1, \ldots, m \text {. }
$$

Besides, the integer $m$ can be bounded by a polynomial in $N$ and $M$ and the matrices $\left\{V_{i}\right\}_{1 \leqslant i \leqslant m}$ satisfy:

$$
\sum_{i=1}^{m} V_{i}^{*} V_{i}=(2 N+7 M+4)^{2} I_{n}
$$

Proof. We denote by $\left\{e_{i}\right\}_{0 \leqslant i \leqslant N+2 M}$ the standard basis vectors in $\mathbb{C}^{N+2 M+1}$. We know from Lemma4.3 that the system (8) admits a solution if and only if there is a pair of non-null vectors $x, y \in \mathbb{C}^{n}$ satisfying

$$
\left\{\begin{array}{l}
x^{\top}\left(e_{0}+p_{i} e_{k_{i}^{1}}+q_{i} e_{k_{i}^{2}}+p_{i} q_{i} e_{N+i}\right) e_{N+M+i}^{\top} y=0, \quad i=1, \cdots, M \\
x^{\top}\left(e_{k_{i}^{1}} e_{k_{i}^{2}}^{\top}-e_{0} e_{N+i}^{\top}\right) y=0, \quad i=1, \cdots, M \\
x^{\top}\left(e_{0}+r_{i} e_{k_{i}^{3}}-e_{N+M+i}\right) e_{j}^{\top} y=0, \quad i=1, \cdots, M, \quad j=0, \ldots, N+2 M \\
x^{\top}\left(e_{i} e_{i}^{\top}-e_{0} e_{0}^{\top}\right) y=0, \quad i=1, \cdots, N+M \\
x^{\top}\left(e_{i} e_{j}^{\top}-e_{j} e_{i}^{\top}\right) y=0, \quad 0 \leqslant i<j \leqslant N+2 M
\end{array}\right.
$$

The system (13) has $N+3 M+(N+2 M+1)(4 M+N) / 2$ bilinear equations. Let $m_{0}=N+3 M+(N+$ $2 M+1)(4 M+N) / 2$ and denote by $\left\{A_{i}\right\}_{1 \leqslant i \leqslant m_{0}}$ the matrices corresponding to the $m_{0}$ bilinear forms 
in (13). Recall that $\left(p_{i}\right)_{i},\left(q_{i}\right)_{i},\left(r_{i}\right)_{i}$ are sequences of numbers in $\{1,-1\}$. Therefore we transformed the system (8) to the following bilinear system:

$$
x^{T} A_{i} y=0, \quad i=1, \ldots, m_{0},
$$

where $A_{i}$ have entries in $\{0,1,-1\}$. We check the five lines in (13) and obtain that

$$
\begin{aligned}
\sum_{i=1}^{m_{0}} A_{i}^{*} A_{i}= & \sum_{i=1}^{M} 4 e_{N+M+i} e_{N+M+i}^{\top}+\sum_{i=1}^{M}\left(e_{k_{i}^{2}} e_{k_{i}^{2}}^{\top}+e_{N+i} e_{N+i}^{\top}\right) \\
& +\sum_{i=1}^{M} \sum_{j=0}^{N+2 M} 3 e_{j} e_{j}^{\top}+\sum_{i=1}^{N+M}\left(e_{i} e_{i}^{\top}+e_{0} e_{0}^{\top}\right) \\
& +\sum_{i<j}\left(e_{j} e_{j}^{\top}+e_{i} e_{i}^{\top}\right)
\end{aligned}
$$

Therefore we have that

$$
\sum_{i=1}^{m_{0}} A_{i}^{*} A_{i}=\left(\begin{array}{cccc}
k_{1} & & & \\
& k_{2} & & \\
& & \ddots & \\
& & & k_{n}
\end{array}\right)
$$

where $k_{i} \leqslant 2 N+7 M+4$ for all $1 \leqslant i \leqslant n$. Remark that due to the third line of equations in (13), for each $0 \leqslant j \leqslant N+2 M$, there is an integer $1 \leqslant n_{j} \leqslant m_{0}$ such that

$$
A_{n_{j}}^{*} A_{n_{j}}=3 e_{j} e_{j}^{\top} \text {. }
$$

By letting $B_{j}=A_{n_{j}} / 3$ we get that:

$$
3 B_{j}^{*} B_{j}=e_{j} e_{j}^{\top} .
$$

For all $1 \leqslant j \leqslant n$ let $l_{j}=(2 N+7 M+4)^{2}-n_{j}$. Let $m=m_{0}+3 \sum_{j=1}^{n} l_{j}$ and $\left\{V_{i}\right\}_{1 \leqslant i \leqslant m}$ be the sequence of matrices containing $\left\{A_{i}\right\}_{1 \leqslant i \leqslant m_{0}}$ and $3 l_{j}$ times the matrix $B_{j}$ for all $1 \leqslant j \leqslant n$. Then we have

$$
\sum_{i=1}^{m} V_{i}^{*} V_{i}=\sum_{i=1}^{m_{0}} A_{i}^{*} A_{i}+\sum_{j=1}^{n} 3 l_{j} B_{j}^{*} B_{j}=(2 N+7 M+4)^{2} I_{n} .
$$

Since for all $1 \leqslant j \leqslant n, B_{j}$ is co-linear to a matrix in $\left\{A_{i}\right\}_{i \leqslant m_{0}}$. The feasibility of the system

$$
x^{T} V_{i} y=0, \quad i=1, \ldots, m
$$

is equal to that of (14). Thus the system (8) admits a solution if and only if there is a nonzero solution to (15).

We now prove Theorem 4.1

Proof. Let $k_{i}^{1}, k_{i}^{2}, k_{i}^{3} \in\{1, \ldots, N\}, p_{i}, q_{i}, r_{i} \in\{ \pm 1\}$ and $k_{i}^{1} \neq k_{i}^{2}$ for all $1 \leqslant i \leqslant M$ such that the system

$$
\left\{\begin{array}{l}
\left(1+p_{i} x_{k_{i}^{1}}\right)\left(1+q_{i} x_{k_{i}^{2}}\right)\left(1+r_{i} x_{k_{i}^{3}}\right)=0, \quad i=1, \cdots, M \\
x_{i}^{2}=1, \quad i=1, \cdots, N
\end{array}\right.
$$

corresponds to an instance of 3SAT problem with $N$ Boolean variables and $M$ clauses. By Lemma 4.4 , we can construct in polynomial time (with respect to $N$ and $M$ ) a sequence of $n \times n$ matrices $\left\{V_{i}\right\}_{1 \leqslant i \leqslant m}$ with entries in $\left\{0, \pm \frac{1}{l}, \pm \frac{1}{3 l}\right\}$ where $l=(2 N+7 M+4)$ such that there is a solution to (16) if and only if there is a nonzero solution to the bilinear system (15). Besides, the matrices $\left\{V_{i}\right\}_{1 \leqslant i \leqslant m}$ satisfy (3).

We deduce the complexity of Problem 2.3 from Theorem 4.1 and Lemma 4.1

Theorem 4.2. Deciding whether a Kraus map is strictly positive (Problem 2.3) is NP-hard.

Remark 4.1. Hillar and Lim [HL13] obtained the NP-hardness of Problem 4.3 by reducing graph 3Colorability problems to it. Let $\left\{W_{1}, \ldots, W_{m}\right\} \subset \mathbb{C}^{n \times n}$ be arbitrary matrices and consider the bilinear system:

$$
x^{T} W_{i} y=0, \forall i=1, \ldots, m \text {. }
$$


Let $U \in \mathbb{C}^{n \times n}$ be any matrix such that

$$
\sum_{i=1}^{m} W_{i}^{*} W_{i}=U^{*} U
$$

If $U$ is not invertible, than the intersection of the null spaces of $\left\{W_{1}, \ldots, W_{m}\right\}$ is not empty and the latter bilinear system is clearly feasible. If $U$ is invertible, than the latter bilinear system is feasible if and only if the following bilinear system is feasible:

$$
x^{T} W_{i} U^{-1} y=0, \forall i=1, \ldots, m .
$$

Hence every instance of Problem 4.3 can be reduced to an instance of Problem 4.1 by computing the matrix $U \in \mathbb{C}^{n \times n}$ satisfying (17). However, in general such a matrix $U$ does not have rational entries. Therefore, it is not obvious to deduce the complexity of Problem 4.1 in the bit model from the NPhardness of bilinear feasibility. In this respect, the proof of Theorem 4.1 should be compared with the one of Hillar and Lim [HL13] proving the latter result. In order to reduce a 3-Colorability problem to a bilinear system, they use cubic roots of the unity to encode the three colors. Some auxiliary variables are also introduced in order to obtain a homogeneous system. However, their construction does not allow to obtain in polynomial time matrices satisfying the constraint (3).

\section{REFERENCES}

[BFS99] Jonathan F. Buss, Gudmund S. Frandsen, and Jeffrey O. Shallit. The computational complexity of some problems of linear algebra. J. Comput. System Sci., 58(3):572-596, 1999.

[BP94] Abraham Berman and Robert J. Plemmons. Nonnegative matrices in the mathematical sciences, volume 9 of Classics in Applied Mathematics. Society for Industrial and Applied Mathematics (SIAM), Philadelphia, PA, 1994. Revised reprint of the 1979 original.

[Den77] E.V. Denardo. Period of connected networks. Math. Oper. Res., 2:20-24, 1977.

[DTDS12] David L. Donoho, Yaakov Tsaig, Iddo Drori, and Jean-Luc Starck. Sparse solution of underdetermined systems of linear equations by stagewise orthogonal matching pursuit. IEEE Trans. Inform. Theory, 58(2):1094-1121, 2012.

[Far96] D. R. Farenick. Irreducible positive linear maps on operator algebras. Proc. Amer. Math. Soc., 124(11):33813390, 1996.

[FHB04] M. Fazel, H. Hindi, and S. Boyd. Rank minimization and applications in system theory. In In American Control Conference, pages 3273-3278. AACC, 2004.

[FSEDS13] Jean-Charles Faugère, Mohab Safey El Din, and Pierre-Jean Spaenlehauer. On the complexity of the generalized MinRank problem. J. Symbolic Comput., 55:30-58, 2013.

[HJ13] Roger A. Horn and Charles R. Johnson. Matrix analysis. Cambridge University Press, Cambridge, second edition, 2013.

[HL13] Christopher J. Hillar and Lek-Heng Lim. Most tensor problems are np-hard. J. ACM, 60(6):45:1-45:39, November 2013.

[LR04] Victor Lomonosov and Peter Rosenthal. The simplest proof of Burnside's theorem on matrix algebras. Linear Algebra Appl., 383:45-47, 2004.

[Nat95] B. K. Natarajan. Sparse approximate solutions to linear systems. SIAM J. Comput., 24(2):227-234, 1995.

[RFP10] Benjamin Recht, Maryam Fazel, and Pablo A. Parrilo. Guaranteed minimum-rank solutions of linear matrix equations via nuclear norm minimization. SIAM Rev., 52(3):471-501, 2010.

[RKW11] David Reeb, Michael J. Kastoryano, and Michael M. Wolf. Hilbert's projective metric in quantum information theory. J. Math. Phys., 52(8):082201, 33, 2011.

[RXH11] Benjamin Recht, Weiyu Xu, and Babak Hassibi. Null space conditions and thresholds for rank minimization. Math. Program., 127(1, Ser. B):175-202, 2011.

[SPGWC10] Mikel Sanz, David Pérez-García, Michael M. Wolf, and Juan I. Cirac. A quantum version of wielandt's inequality. IEEE Trans. Inf. Theor., 56(9):4668-4673, September 2010.

[SSR10] Rodolphe Sepulchre, Alain Sarlette, and Pierre Rouchon. Consensus in noncommutative spaces. In Proceedings of the 49th IEEE Conference on Decision and Control, pages 6596-6601, Atlanta, USA, Dec 2010.

INRIA AND CMAP, École Polytechnique. 91128 PALAiseau CEDEX

E-mail address: Stephane.Gaubert@inria.fr

School of Mathematics, University of Edinburgh, Edinburgh, EH9 3JZ, UK

E-mail address: zheng.qu@ed.ac.uk 